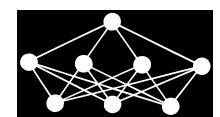

\title{
PATH MULTIMODALITY IN A FEEDFORWARD SNN MODULE, USING LIF WITH LATENCY MODEL
}

\author{
G. Susi, A. Cristini $\dagger$ M. Salerno*
}

\begin{abstract}
In this paper, the network transmission properties of a feedforward Spiking Neural Network (SNN) affected by synchronous stimuli are investigated with respect to the connection probability and the synaptic strengths. By means of an event-driven method, all simulations are conducted using the Leaky Integrateand-Fire with Latency (LIFL) model. Typical cases are taken into consideration, in which a network section (module) is able to process the input information, introducing a particular behavior, that we have called path multimodality. Simulation results are discussed. Through this phenomenon, the output layer of the network can generate a number of temporally spaced groups of synchronous spikes. The multimodality effect could be applied for various purposes, for instance in coding or else transmission issues.
\end{abstract}

Key words: Feedforward SNN, LIF with latency model, synchrony, firing rate, path multimodality

Received: December 20, 2013

DOI: $10.14311 /$ NNW.2016.26.021

Revised and accepted: June 17, 2016

\section{Introduction}

Spiking Neural Networks (SNNs) represent a class of biological inspired neural networks that are widely implemented in many research fields, such as engineering, neurophysiology, neuroscience. These kind of networks seem very useful, in particular, for the elaboration of both sensory and cognitive information $[2,3,15,23,27]$. For example, the transmission of the spiking activity through a neural network appears of great interest and many computational models have been proposed to approach this problem, such as feedforward models with convergent-divergent connections $[1,8,11,16-18,20,22,28,34,35]$. These studies involve rate codes or else temporal codes $[24,27]$, depending on the spiking activity which can be asynchronous, or else synchronous [21]. This difference is due to the necessity of saving the information in subsequent stages of a sensory pathway.

\footnotetext{
*Gianluca Susi, Mario Salerno University of Rome Tor Vergata, Via del Politecnico 1, 00133 , Rome, Italy, E-mail: gianluca.susi@uniroma2.it, mario.salerno@uniroma2.it

${ }^{\dagger}$ Alessandro Cristini - Corresponding author, University of Rome Tor Vergata, Via del Politecnico 1, 00133, Rome, Italy, E-mail: alessandro.cristini@uniroma2.it
} 
In these studies, Leaky Integrate-and-Fire (LIF) models are typically used. Their low computational cost allows the properties of large spiking networks to be investigated, maintaining some important biological neuronal features (i.e., subthreshold decay, synaptic integration and spiking threshold) [4,5].

The information propagation depends on the network parameters. In fact, it was shown that feedforward networks with low probability of shared connections and strong synaptic strengths permit a faithful transmission of asynchronous firing rate. In the opposite case, a transmission of synchrony is allowed [21].

In the paper, simulation results of a feedforward SNN affected by synchronous stimuli are discussed. The network transmission properties are investigated in function of the connection probabilities and the synaptic strengths. Furthermore, all simulations are conducted on a LIF with Latency (LIFL) model, simulated through an event-driven method. The latency phenomenon introduces a nonlinearity in the suprathreshold neuron behavior, allowing the information propagation in a multimodal manner through consecutive layers of a feedforward neural network (FNN). It will be shown how the latency property affects synchronous inputs to a neuronal layer, and causes temporal clusters for the spike generated by the layer itself. Then, the typical behaviors that occur under certain hypothesis will be classified, and will be explained how the temporal distance of the neuronal groups depends on the network parameters. With the aim to distinguish this kind of multimodality from those already defined in literature $[32,33]$, this effect will hereinafter be called path multimodality. Taking into account the fact that synchronous spikes are provided to the network input, the path multimodality represents the capability of a network section (in this case a module) of generating a number of temporally spaced groups of synchronous spikes at the output network. Perfect synchronous input may be thought as spike generated by a previous FNN module in jitter recovery condition [7]. Note that it has been highlighted how the integration of a large number of Gaussian-distributed inputs with small amplitude (i.e., high synaptic convergence) produces a substantial reduction of the dispersion in the arrival times of such inputs, or input jitter, namely the standard deviation of the inputs. Indeed, the dispersion of the spike timings on a target neuron, or output jitter (i.e., the standard deviation of the outputs) can be less than the input jitter [6]. Thus, it is relevant to understand how this synchronous group can propagate toward downstream modules.

\section{Theory and methods}

\section{$2.1 \quad$ Neuron model}

In this work, a simple spiking neuron model with features similar to those of the classic LIF is introduced [4]. The main difference from the LIF model is the presence of an expression, called firing equation, which qualitatively describes the neuron behavior in the suprathreshold region: when the membrane potential $S$ reaches the spiking threshold, the firing is not instantaneous, but it occurs after a variable continuous time delay $t_{\mathrm{f}}$, called latency [12]:

$$
t_{\mathrm{f}}=\frac{1}{S-1} \text {. }
$$


Susi G., Cristini A., Salerno A.: Path multimodality in a Feedforward SNN...

In Eq. (1), the relationship between the time delay $t_{\mathrm{f}}$ and the membrane potential $S$ is a branch of rectangular hyperbola (Fig. 1) [30]. This is an approximation of the behavior of the real biological phenomenon and it has been obtained by simulating a patch of neuronal membrane stimulated with short current pulses, solving the Hodgkin-Huxley equations [14] by means of the NEURON simulator [13].

In this model, normalized real and non-negative quantities are considered. Thus, the variable $S$ indicates the inner state of the neuron, and the variable $t_{\mathrm{f}}$, called time-to-fire, can be linked to the spike latency. In addition, the spiking threshold $S_{\mathrm{th}}=1+d$ is introduced, where $d$ denotes the threshold constant. The latter quantity has been chosen in order to have a finite maximum value for the time-to-fire, $t_{\mathrm{f}, \max }=1 / d$. When $S=0$ the neuron is in its resting state.

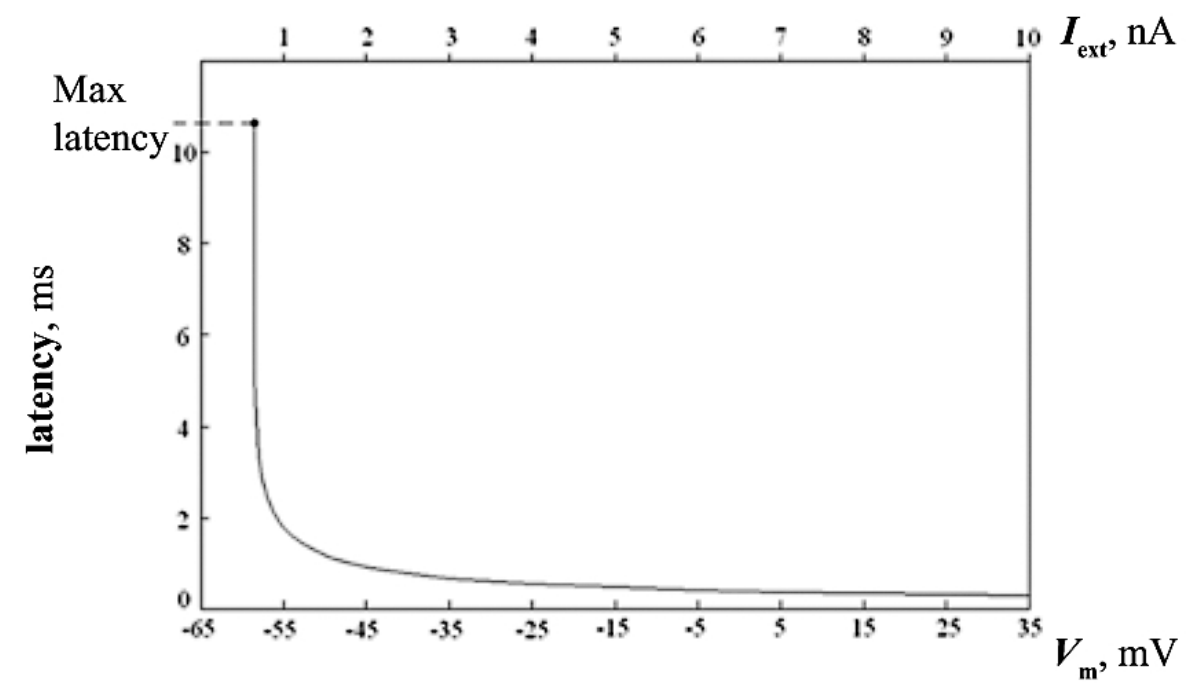

Fig. 1 Latency as a function of the membrane potential $V_{\mathrm{m}}$ or else of the current amplitude $I_{\mathrm{ext}}$, equivalently. Note that $V_{\mathrm{m}}$ is the biological counterpart of the inner state $S$ of our model, whereas, $I_{\mathrm{ext}}$ is the biological counterpart of the presynaptic weight $P_{\mathrm{r}}$ of our model. The curve has been obtained simulating a patch of cell membrane solving the Hodgkin-Huxley equations using NEURON environment.

Taking into account that each neuron receives synaptic inputs from a large number of synapses (synaptic convergence) and propagates spikes to a high number of neurons (synaptic divergence), the quantity $P_{\mathrm{r}}$, presynaptic weight, denotes the signal transmitted (i.e., single pulses) from one neuron to a number of other neurons. Finally, the quantity $P_{\mathrm{w}}$, postsynaptic weight, is associated to each connection, indicating the strength between a couple of neurons. For the sake of convenience, the weights are chosen to be constant, then not subjected to synaptic plasticity [9], and arbitrarily assigned between 0 and a maximum value. Moreover, if $P_{\mathrm{w}}$ is equal to 0 , the related connection is not present. The following equations describe the rules for the state updating, in passive mode (subthreshold region) and in active mode (suprathreshold region), respectively (Fig. 2): 


$$
\begin{gathered}
S=S_{\mathrm{p}}+P_{\mathrm{r}} P_{\mathrm{w}}-L_{\mathrm{d}} \Delta t, \text { for } S<S_{\mathrm{th}}, \\
S=S_{\mathrm{p}}+P_{\mathrm{r}} P_{\mathrm{w}}, \text { for } S \geq S_{\mathrm{th}} .
\end{gathered}
$$

In Eqs. (2)-(3), $S_{\mathrm{p}}$ represents the previous state, $L_{\mathrm{d}}$ the subthreshold linear decay, and $\Delta t$ is the temporal difference between two consecutive incoming spikes. When $S \geq S_{\text {th }}$ (suprathreshold region), the neuron becomes active and it is ready to fire, but it remains still sensitive to incoming spikes. In this region, for each new state the bijective relationship (1) is evaluated. Thus, as times advances and new spikes arrive, the state $S$ is properly increased (decreased), due to the excitatory (inhibitory) effect, and $t_{\mathrm{f}}$ is evaluated [31]. When the last computed $t_{\mathrm{f}}$ expires, a spike is generated, the inner state becomes $S=0$ (reset) and the neuron remains insensitive for a time called the absolute refractory period $\left(t_{\mathrm{arp}}\right)$, like in the case of biological neurons.

Due to the necessity of simulating the latency effect, the use of an event-driven approach for the simulation of the spiking neural network is required. Moreover, this simulation technique let to investigate large network properties with high precision, requiring a low computational cost $[10,25,29]$.

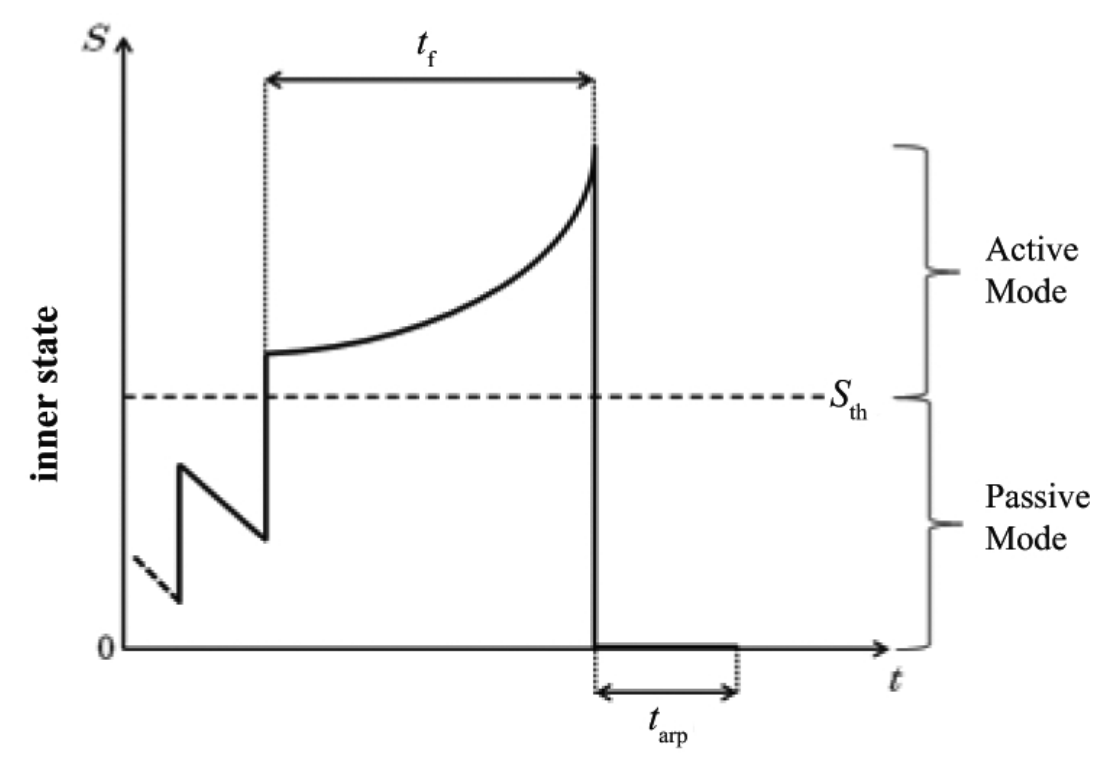

Fig. 2 Passive and Active Mode. $S_{\mathrm{th}}$ indicates the spiking threshold.

\subsection{Latency effect on the behavior of a FNN module}

Defining a module as a network section that is able to introduce particular delays, a feedforward module without axonal delays and affected by a synchronous vertical input train is considered here (Fig. 3). At each simulation, the incoming spikes have the same amplitude (i.e., $P_{\mathrm{r}}$ is chosen to be constant and $P_{\mathrm{w}}$ is fixed by (4), 
see Section 3). Thanks to the presence of the latency in the neuron model, the information through the network can be transmitted in an asynchronous manner to the output (Fig. 4). The particular behavior of the transmission mainly depends on both postsynaptic weights and the connection probability. As an example, in the case of a sparsely connected network with high postsynaptic weight values, an asynchronous pulse train is generated (Fig. 3).

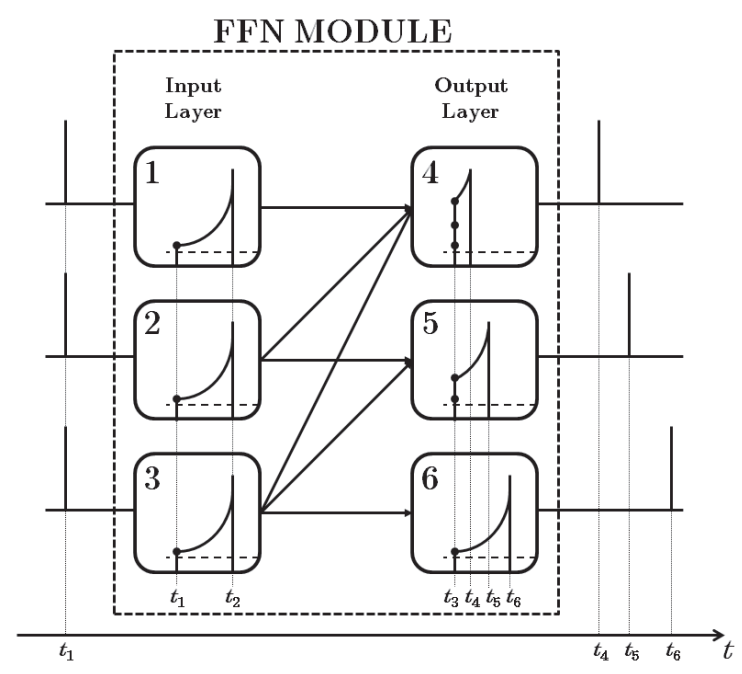

Fig. 3 An example of a Feedforward Module Topology (FMT) composed by six LIFL neurons. It is shown a case of sparsely connected network. At $t_{1}$ three synchronized inputs arrive to the input layer. The magnitude of these inputs is sufficient to provoke an initial active state $\left(S_{\mathrm{ia}}\right)$ greater than the threshold. Then, neurons 1 , 2 and 3 fire at $t_{2}$. Since there is no axonal delay, the spikes arrive to the output layer at $t_{3}=t_{2}$. Because of each neuron has a different fan-in, their firings are asynchronous (i.e., $t_{4}, t_{5}, t_{6}$ are not synchronous, depending on the related $S_{\mathrm{ia}}$ ).

In the present work, a double layer feedforward network composed by 50 neurons per layer is used to carry out considerations about the multimodality effect (Fig. 5). To this purpose, the connections characterization, between the two layers, is realized by means of the two parameters $P_{\mathrm{w}}$ and CF (Connection Factor). Note that the number of neurons per layer is relatively small because only a very small portion of neurons in the brain responds to a stimulus (sensory or cognitive stimuli) [21]. Typically tens or few hundreds of neurons per layer are considered. The simulation procedure is shown in Section 3.

\subsection{Path multimodality}

As mentioned in the introduction, the path multimodality is the capability of a network path (a feedforward module, in this particular study) of generating in the output more groups of synchronous spikes temporally spaced, when it is stimulated by a synchronous input train. If the number of neurons in a FMT grows up, the phenomenon described above is more evident. Indeed, under particular conditions, 


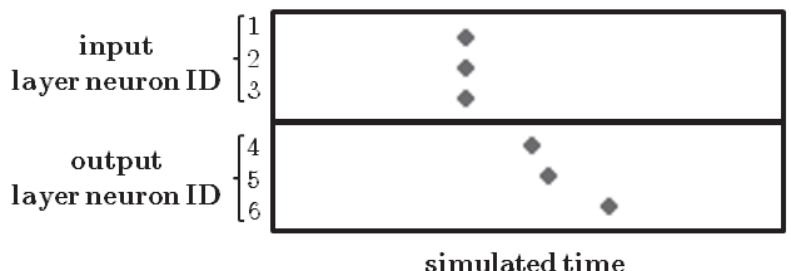

simulated time

Fig. 4 Raster plot of a simulation made using the module shown in Fig. 3. It is reported the temporal behavior of the FMT. On the vertical axis the neuron number is denoted. On the horizontal axis the simulated time is indicated.

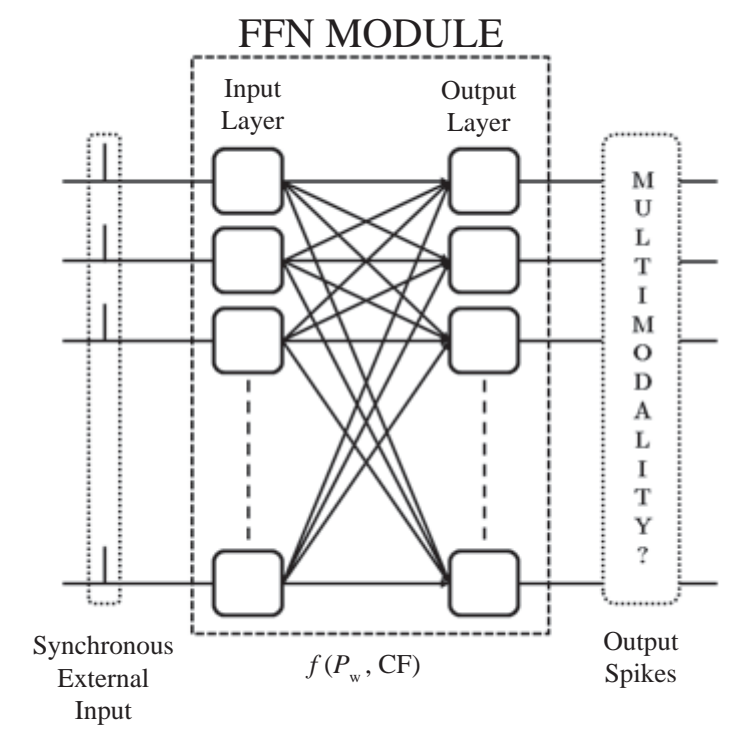

Fig. 5 Input: external sources that generate synchronous spikes. Input Layer and Output Layer represent the feedforward network module; the connections between the layers can be both fully and sparsely connected. The output behavior is analyzed in function of $P_{\mathrm{w}}$ and CF. Finally, the kind of multimodality is classified.

this behavior is replicated, so that, in the output of a module, some temporal groups composed by synchronous spikes can be obtained. Depending on the network parameters, these spike timing group intervals can present regular or else irregular distributions. The purpose of the present work is to investigate this phenomenon and the behaviors that could be obtained varying some network parameters.

In order to reduce the freedom degrees in the simulation process, constant and identical $P_{\mathrm{w}}$ values are assigned for the connections between the two layers, as shown in Section 3, so that most of the output spikes are generated. In this way, defining the neuron fan-in as the number of the incoming connections, when the module receives only synchronous inputs, the output spike timing distribution is uniquely related to the fan-in configuration of the output layer neurons. On the 
Susi G., Cristini A., Salerno A.: Path multimodality in a Feedforward SNN...

basis of the above described conditions, the number of the possible modes of a FMT is equal to the number of the different fan-in values concerning the output layer (Fig. 6).

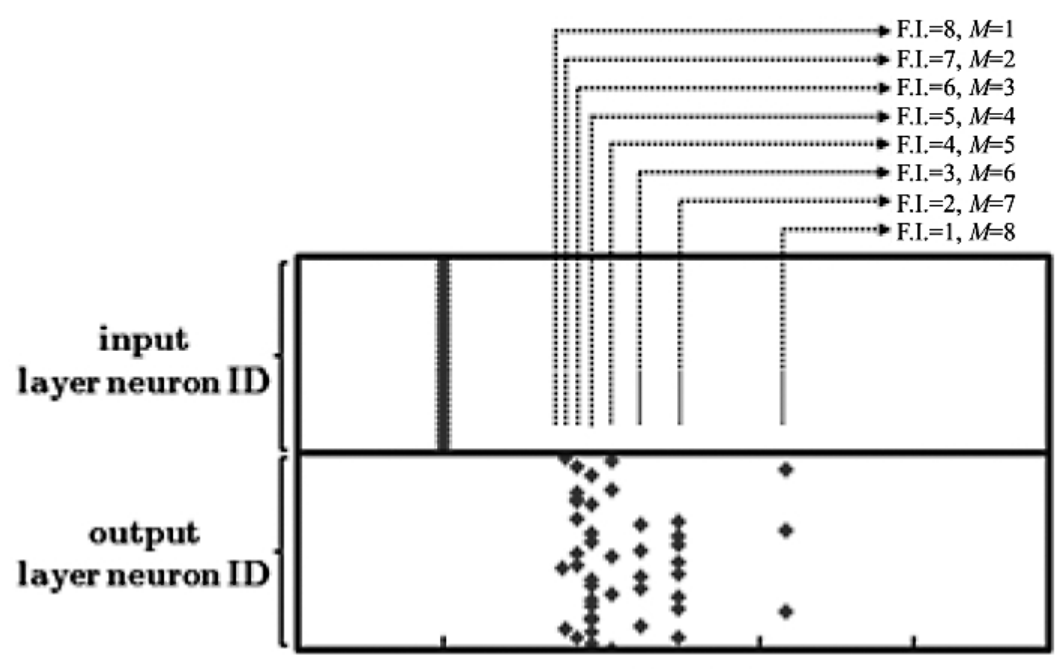

simulated time

Fig. 6 Raster plot of a typical simulation. The eight spike timing groups emerged after the stimulation are indicated with the related fan-in (F.I.) and the corresponding elicited latency, or mode (M).

Taking into account the above described network conditions and neuron features, it seems quite easy to imagine how a neuron model, that does not implement the latency phenomenon, does not allow different spike timings intervals to be generated through the network. Then, the introduction of the latency in the model allows generating output spikes with intervals related to the network parameters.

In general, depending on which input layer neuron is excited, it is easy to understand how the output can be characterized by some modes rather than other ones. In addition, the discussion becomes more complex if a real scenario is considered, in which other conditions could be present: asynchronous input spikes, variable input spike amplitude, variable connection weights $P_{\mathrm{w}}$ and axonal delays. In future works, these conditions will be introduced in order to extend the present discussion.

\section{Simulation results}

In this section, in order to study the behavior of the network under proper conditions, both model and network parameters are introduced. Also, the simulation results are presented. There are two basic parameters: postsynaptic weight and connection factor. The fist one is defined by the following relationship:

$$
P_{\mathrm{w}}=\frac{S_{\mathrm{th}}}{(N \times R)},
$$


where $S_{\text {th }}$ represent the spiking threshold (conventionally fixed to 1.04), $N$ is the number of the active connections and $R$ is the neuronal threshold ratio. The latter can be expressed by the relationship:

$$
R=\frac{S_{\mathrm{th}}}{S_{\mathrm{ia}}},
$$

in which $S_{\mathrm{ia}}$ is the initial active state (as indicated in Fig. 3) reached by an output neuron, thanks to the overall incoming spikes. The values of $R$ have been chosen in the range $[0.1,0.9]$ with step equal to 0.1 . The expressions (4)-(5) have been obtained on the basis of similar relationships discussed in Burkitt and Clark [6]. In addition, the connection factor is defined as

$$
\mathrm{CF}=\frac{N}{N_{\text {tot }}},
$$

as mentioned above, $N$ represents the number of the active connections and $N_{\text {tot }}$ is the total number of the connections. In the case of fully connected network $\mathrm{CF}$ is equal to 1 . Note that, in order to obtain values of $\mathrm{CF}$ in the range $[0.1$, 1], the connections are chosen in a pseudorandom manner. The results, shown in the following raster plots, have been obtained by simulating a network composed of 2 layers: the first one, input layer, provides synchronized inputs. The second one, output layer, is connected to the input layer with connections having a certain connection factor. Therefore, the network can be sparse (i.e., $0<\mathrm{CF}<1$ ) or else fully (i.e., $\mathrm{CF}=1$ ) connected. The simulations have been performed by varying $P_{\mathrm{w}}$ (i.e., $R$ and $N$ ) and $\mathrm{CF}$ (i.e., $N$ ).

On the basis of the nonlinearity of the latency curve (Fig. 1), it can be possible to notice substantially different output spike timing distribution behaviors. Indeed, depending on the portion of the curve affected by the $P_{\mathrm{w}}-\mathrm{CF}$ combination, they can be essentially grouped in three typical trends:

- Non-constant behavior. Due to the fan-in decreasing of the target neuron (i.e., the decreasing of the number of the incoming connections), this behavior is characterized by an irregular increasing of the spike timing distance between the synchronous groups. In this case, the spike timing groups are irregularly distributed (see Fig. 7).

- Quasi-constant behavior. It is characterized by similar intervals among synchronous groups. In this case, the spike timing groups are quite regularly distributed (see Fig. 8).

- Perfect-Synchrony behavior. It is characterized by a single spike timing group. This emerges only in the case of $\mathrm{CF}=1$ (see Fig. 9).

In all cases, thanks to the latency effect, it is possible to identify several activated synchronous groups. Depending on the neuronal and network parameters, these groups can be temporally distributed in quasi-constant or non-constant manner. Then, depending on the network parameters, it is possible to generate pulse trains with both irregular or else regular spike timing group distribution, even in 
Susi G., Cristini A., Salerno A.: Path multimodality in a Feedforward SNN...

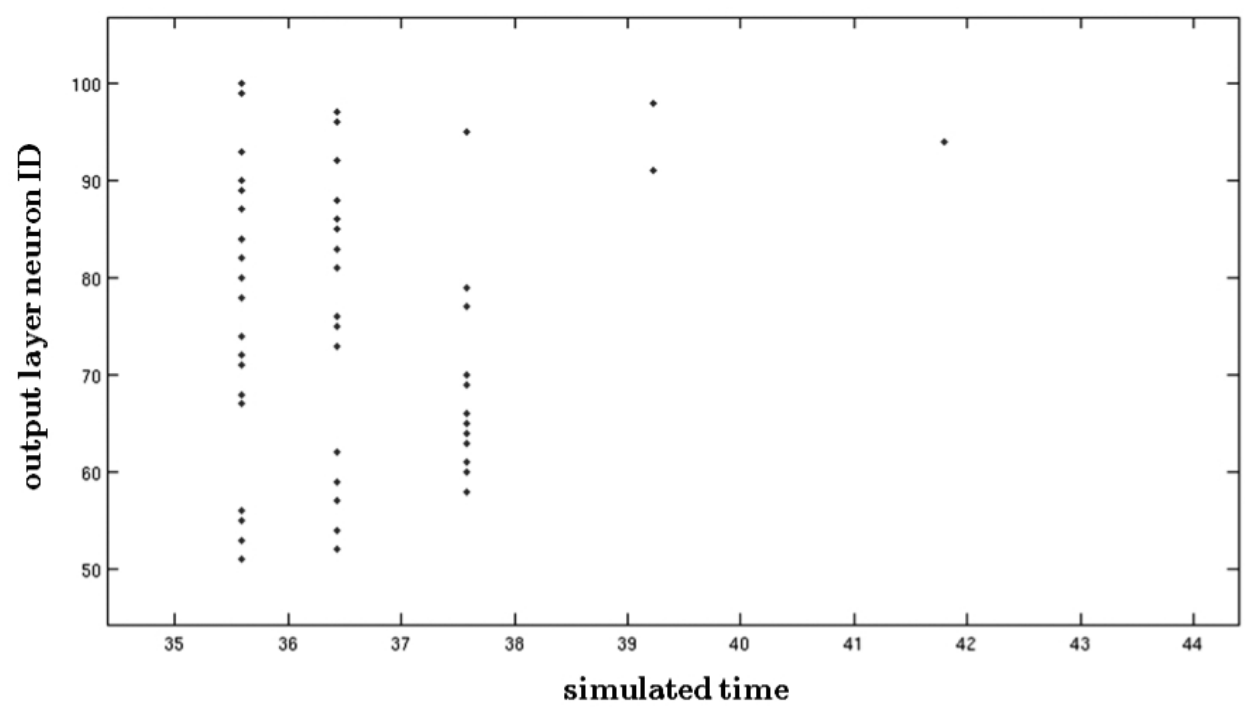

Fig. 7 Raster plot that shows non-constant behavior. In this simulation fan-in equal to 50, 49, 48, 47, 46 for the output neurons have been observed.

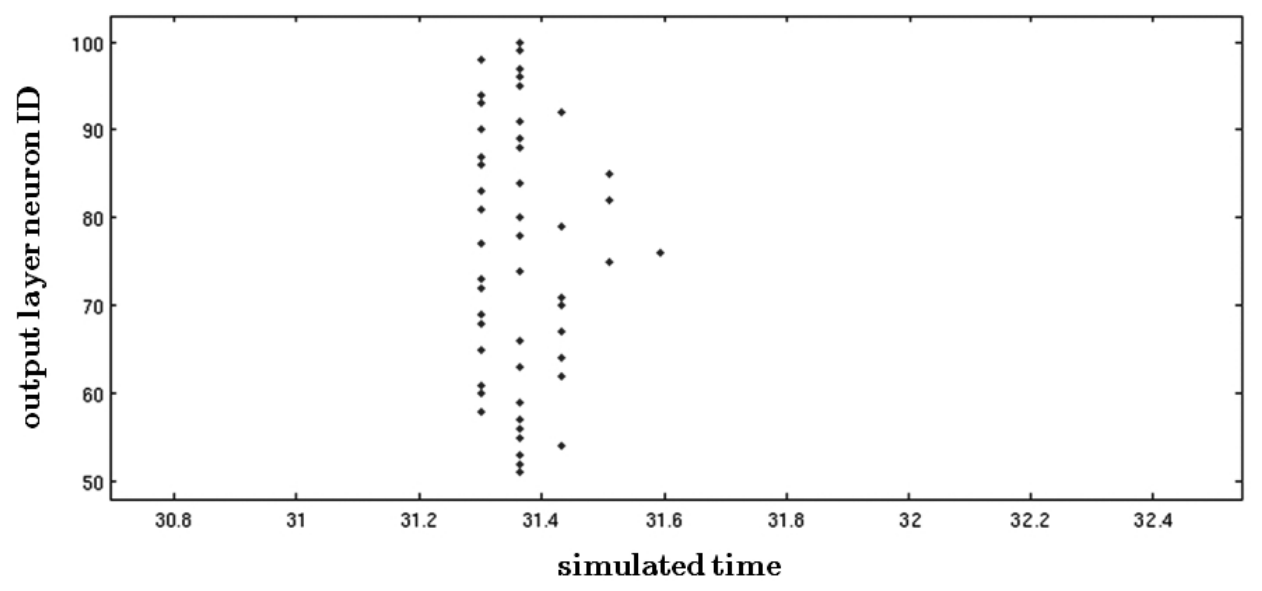

Fig. 8 Raster plot that shows quasi-constant behavior. In this simulation fan-in equal to 50, 49, 48, 47, 46 for the output neurons have been observed.

the case of synchronous input spikes. Note that, only in the case of $\mathrm{CF}=1$, the firings of the output layer are generated in a perfect-synchrony manner. Furthermore, the more both synaptic weights and $\mathrm{CF}$ are high (i.e., high $P_{\mathrm{w}}$ and low $R$ values), the more the spike timing groups appear close in time. Finally, for $\mathrm{CF}$ increasing, the number of synchronous groups will decrease, up to the limit value of $\mathrm{CF}=1$. In this case, only a synchronous group is present (as shown in Fig. 9).

The network analysis has been performed taking into account the Coefficient of Variation, CV. The latter is a measure of the dispersion in the ISI (Inter-Spike 


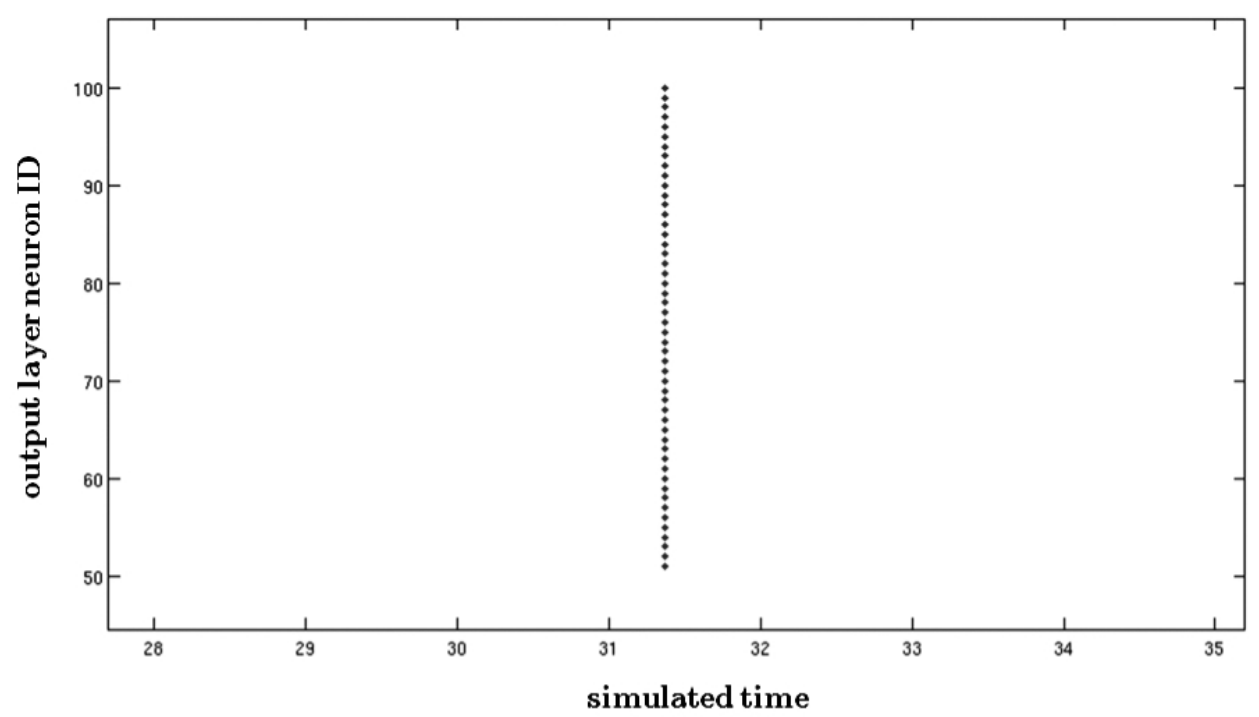

Fig. 9 Raster plot that shows perfect-synchrony behavior. The fan-in of the output neurons is equal to 50 for each neuron.

Interval) distribution, and it is computed by means of the following relationship:

$$
\mathrm{CV}=\sqrt{\frac{1}{N-2} \sum_{i=1}^{N-1}\left(\Delta t_{i}-\overline{\Delta t}\right)} / \overline{\Delta t}
$$

in which $N$ is the total number of spikes, $\Delta t$ is a given ISI, and $\overline{\Delta t}$ is defined as

$$
\overline{\Delta t}=\frac{1}{N-1} \sum_{i=1}^{N-1} \Delta t .
$$

The coefficient of variation can be computed either for a single neuron or the entire network [19]. We have considered the latter case for our study. Note that for a constant $\Delta t$ the $\mathrm{CV}$ is zero, whereas it is greater than 1 (for example) when the distribution of spike intervals become hyperexponential [26].

Since we have performed 100 trials for each combination of $\mathrm{CF}-R$, mean and standard deviation of CV over all trials have been calculated.

As above discussed, for high synaptic weights (then low values of $R$ ) and low values of shared connections (i.e., low values of $\mathrm{CF}$ ), $\mathrm{CV}$ is greater than 1 (red regions in the most left colormap in Fig. 10), implying non-constant behaviors. On the other hand, CV approaches to zero (blue regions in the most left colormap in Fig. 10) for high values of the shared connections and high values of the synaptic weights. Finally, in the case of $\mathrm{CF}=1$ the coefficient of variation can not be defined because there is only one synchronous group (i.e., perfect-synchrony behavior), and then for this value the colormap is blank.

Another observation is that $\mathrm{CV}$ is quite independent of the subthreshold decay $L_{\mathrm{d}}$. Indeed, the behaviors exhibited from the module are insensitive to the 
Susi G., Cristini A., Salerno A.: Path multimodality in a Feedforward SNN...

variation of the parameter $L_{\mathrm{d}}$. This happens because in this study the inputs are synchronous, then the state of the target neurons (i.e., the neurons of the output layer) are not able to show the leaky behavior.

\section{Conclusions}

In this paper, the case of feedforward spiking neural networks stimulated by synchronous inputs is analyzed. Under proper working conditions, the chance of the generation of groups of synchronous spikes is investigated.

In order to obtain quite biological plausible dynamics, a Leaky Integrate-andFire with Latency model is considered. On the basis of the nonlinearity introduction in the neuron model, new kind of behaviors can emerge in a simple spiking FNN module, in which the generation of some synchronous spike timing groups is possible.

In this work a single FNN module has been taken into consideration, in order to study the simplest case of input-output behavior resulting from the pathmultimodality effect. As mentioned in the introduction, there are conditions that allow asynchronous propagation and other conditions that allow synchronous propagation. In this work, we focused our study on the simple case of synchronous inputs, showing how the latency phenomenon can affect the behavior of the network for the generation of the output spike sequence. For this purpose, typical cases are investigated by varying the parameters of the model, showing how a network section is able to process the input information, highlighting a particular phenomenon, called path multimodality. Depending on the distribution of spike timing groups, different trends have been classified: non-constant, quasi-constant, and perfect-synchrony behaviors. Each case has been related to combinations of both network and neuron parameters, as shown in Fig. 10. Note that, the response time behavior of the
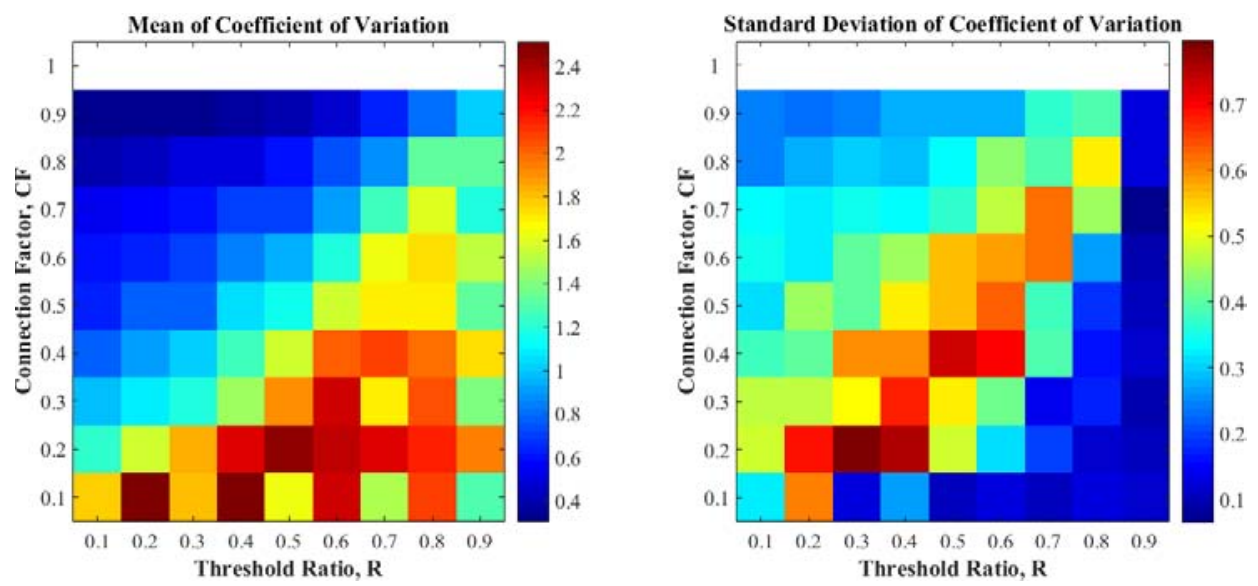

Fig. 10 Mean and standard deviation of the Coefficient of Variation for the case $L_{\mathrm{d}}=0.001$. 


\section{Neural Network World 4/2016, 363-376}

neuron depends basically on the amount of stimulation (see Eqs. (1), (2), (3)), and then on the portion of latency curve affected by the stimuli. Finally, a mapping of the behaviors varying the model parameters has been provided.

The presented work represents a first contribution toward the modular study of complex topologies considering the path multimodality effect. Taking into account that a feedforward network is composed by several modules, the effect of multimodality can be considered for different scenarios, e.g., in coding or else transmission. In the synthesis phase, this effect can be considered in order to obtain particular rhythms by means of a multi-modular FNN architecture. On the other hand, in the analysis phase, this effect can be considered in order to infer the network architecture starting from a given response in terms of spike trains. Of course, further efforts will be made to investigate in details all the opportunities offered by the multimodality effect. In future works, further conditions will be considered, such as input spikes with variable amplitude (i.e., not fixed presynaptic weights $P_{\mathrm{r}}$ ), postsynaptic weights $P_{\mathrm{w}}$ affected by synaptic plasticity and axonal delays as well.

\section{References}

[1] AERTSEN A., DIESMANN M., GEWALTIG M.O. Propagation of synchronous spiking activity in feedforward neural networks. J. Physiol.-Paris. 1996, 90(3-4), pp. 243-247, doi: $10.1016 / \mathrm{S} 0928-4257$ (97) 81432-5.

[2] BELATRECHE A., MAGUIRE L.P., McGINNITY M. Advances in design and application of spiking neural networks. Soft Computing - A Fusion of Foundations, Methodologies and Applications. 2006, 11(3), pp. 239-248, doi: 10.1007/s00500-006-0065-7.

[3] BRETTE R., RUDOlPH M., CARNEVAlE T., HINES H., BEEMAN D., BOWER J.M., DIESMANN M., MORRISON A., GOODMAN P.H., JR HARRIS F.C., ZIRPE M., NATSCHLIÄGER T., PECEVSKI D., ERMENTROUT B., DJURFELDT M., LANSNER A., ROCHEL O., VIEVIlle T., MUlleR E., DAVISON A.P., EL BOUSTANI S., DESTEXHE A. Simulation of networks of spiking neurons: A review of tools and strategies. J. Comput. Neurosci. 2007, 23(2), pp. 349-398, doi: 10.1007/s10827-007-0038-6.

[4] BURKITT A.N. A review of the integrate-and-fire neuron model: I. Homogeneous synaptic input. Biol. Cybern. 2006, 95(1), pp. 1-19, doi: 10.1007/s00422-006-0068-6.

[5] BURKITT A.N. A review of the integrate-and-fire neuron model: II. Inhomogeneous synaptic input and network properties. Biol. Cybern. 2006, 95(2), pp. 97-112, doi: 10.1007/ s00422-006-0082-8.

[6] BURKITT A.N., CLARK G.M. Analysis of integrate-and-fire neurons: synchronization of synaptic input and spike output. Neural Comput. 1999, 11(4), pp. 871-901, doi: 10.1162/ 089976699300016485.

[7] CARDARILli G.C., CRISTINI A., DI NUNZIO L., RE M., SALERNO M., SUSI G. Spiking neural networks based on LIF with latency: Simulation and synchronization effects. In: IEEE Proc. of the 2013 Asilomar Conference on Signals, Systems and Computers. Pacific Grove, CA, USA, 2013, pp. 1838-1842.

[8] CÂTEAU H., FUKAI T. Fokker-Planck approach to the pulse packet propagation in synfire chain. Neural Netw. 2001, 14(6-7), pp. 675-685, doi: 10.1016/S0893-6080(01)00065-X.

[9] CITRI A., MALENKA R.C. Synaptic plasticity: multiple forms, functions, and mechanisms. Neuropsychopharmacology. 33(1), 2008, pp. 18-41, doi: 10.1038/sj.npp.1301559.

[10] D'HAENE M., SCHRAUWEN B., VAN CAMPENHOUT J., STROOBANDT D. Accelerating Event-Driven Simulation of Spiking Neurons with Multiple Synaptic Time Constants. Neural Comput. 2009, 21(4), pp. 1068-1099, doi: 10.1162/neco.2008.02-08-707. 
Susi G., Cristini A., Salerno A.: Path multimodality in a Feedforward SNN...

[11] DIESMANn M., GEWALTiG M.O., AERTSEN A. Stable propagation of synchronous spiking in cortical neural networks. Nature. 1999, 402(6761), pp. 529-533, doi: 10.1038/990101.

[12] FITZHUGH R. Mathematical models of threshold phenomena in the nerve membrane. Bull. Math. Biophys. 1955, 17(4), pp. 257-278, doi: 10.1007/BF02477753.

[13] HINES M.L., CARNEVALE N.T. The NEURON simulation environment. Neural Comput. 1997, 9(6), pp. 1179-1209, doi: 10.1162/neco.1997.9.6.1179.

[14] HODGKIN A.L., HUXLEY A.F. A quantitative description of membrane current and application to conduction and excitation in nerve. J. Physiol. 117(4), pp. 500-544, 1952.

[15] IZHIKEVICH E.M. Which Model to Use for Cortical Spiking Neurons? IEEE Trans. on Neural Networks. 2004, 15(5), pp. 1063-1070, doi: 10.1109/TNN.2004.832719.

[16] JAHNKE S., MEMMESHEIMER R.-M., TIMME M. Guiding synchrony through random networks. Phys. Rev. 2012, 2(041016), pp. 1-12, doi: PhysRevX.2.041016.

[17] JAHNKE S., MEMMESHEIMER R.-M., TIMME M. Propagating synchrony in feed-forward networks. Front. Comput. Neurosci. 2013, 7(153), pp. 1-25, doi: 10.3389/fncom.2013.00153.

[18] KISTLER W.M., GERSTNER W. Stable propagation of activity pulses in populations of spiking neurons. Neural Comput. 2002, 14(5), pp. 987-997, doi: 10.1162/ 089976602753633358.

[19] KUEBLER E.S., THIVIERGE J.-P. Spiking variability: Theory, measures, and implementation in MATLAB. Quantitative Methods for Psychology. 10(2), 2014, pp. 131-142.

[20] KUMAR A., ROTTER S., AERTSEN A. Conditions for propagating synchronous spiking and asynchronous firing rates in a cortical network model. J. Neurosci. 2008, 28(20), pp. 5268-5280, doi: 10.1523/JNEUROSCI. 2542-07.2008.

[21] KUMAR A., ROTTER S., AERTSEN A. Spiking activity propagation in neuronal networks: Reconciling different perspectives on neural coding. Nature Reviews Neuroscience. 2010, 11, pp. $615-627$, doi: $10.1038 / \mathrm{nrn} 2886$.

[22] LITVAK V., SOMPOLINSKY H., SEGEV I., ABELES M. On the transmission of rate code in long feedforward networks with excitatory-inhibitory balance. J. Neurosci. 2003, 23(7), pp. 3006-3015.

[23] MAASS W. Networks of spiking neurons: The third generation of neural network models. Neural Netw. 1997, 10(9), pp. 1659-1671, doi: 10.1016/S0893-6080(97)00011-7.

[24] MAASS W. Paradigms for computing with spiking neurons. In: Models of Neural Networks, ed. New York: Springer-Verlag, (Eds). 2002, pp. 373-402, doi: 10.1007/ 978-0-387-21703-1_9.

[25] MATTIA M., DEL GIUDICE P. Efficient event-driven simulation of large networks of spiking neurons and dynamical synapses. Neural Comp. 2000, 12(10), pp. 2305-2329, doi: 10.1162/ 089976600300014953.

[26] PIPA G., GRÜN S. Impact of spike train autostructure on probability distribution of joint spike events. Neural Comput. 2013, 25(5), pp. 1123-1163, doi: 10.1162/NECO_a_00432.

[27] PONULAK F., KASIŃSKI A. Introduction to spiking neural networks: Information processing, learning and applications. Acta Neurobiol Exp. 2011, 71(4), pp. 409-433.

[28] RATTÉ S., HONG S., DE SCHUTTER E., PRESCOTT S.A. Impact of neuronal properties on network coding: roles of spike initiation dynamics and robust synchrony transfer. Neuron. 2013, 78(5), pp. 758-772, doi: 10.1016/j.neuron.2013.05.030.

[29] ROS E., CARRILlO R., ORTIGOSA E.M., BARBOUR B., AGÍS R. Event-Driven Simulation Scheme for Spiking Neural Networks Using Lookup tables to Characterize Neuronal Dynamics. Neural Comput. 2006, 18(12), pp. 2959-2993, doi: 10.1162/neco.2006.18.12.2959.

[30] SALERNO M., SUSI G., CRISTINI A. Accurate Latency Characterization for Very Large Asynchronous Spiking Neural Networks. In: Proc. of the 4th Int. Conf. on Bioinformatics Models, Methods and Algorithms, 2011, pp. 116-124.

[31] SAlERno M., SUSI G., CRISTINI A., SANFELICE Y., D'ANNESSA A. Spiking Neural Networks As Continuous-Time Dynamical Systems: Fundamentals, Elementary Structures And Simple Applications. ACEEE Int. J. on Information Technology. 2013, 3(1), pp. 80-89, doi: 01.IJIT.3.1.1129. 


\section{Neural Network World 4/2016, 363-376}

[32] TAHMASEBI P., HEZARHANI A. Application of a Modular Feedforward Neural Network for Grade Estimation. Neural Resources Research. 2011, 20(1), pp. 25-32, doi: 10.1016/j . cageo. 2012.02.004.

[33] TORRES-SOSPEDRA J., HERNÁNDEZ-ESPINOZA C., FERNÁNDEZ-REDONDO M. Designing a New Multilayer Feedforward Modular Network for Classification Problems. In: The 2013 IEEE Int. Joint Conf. on Neural Networks Proc. 2006, pp. 1284-1289.

[34] VAN ROSSUM M.C., TURRIGIANO G.G., NELSON S.B. Fast propagation of firing rates through layered networks of noisy neurons. J. Neurosci. 2002, 22(5), pp. 1956-1966.

[35] VOGELS T.P., ABBOTT L.F. Signal propagation and logic gating in networks of integrateand-fire neurons. J. Neurosci. 2008, 25(46), pp. 10786-10795, doi: 10.1523/JNEUROSCI. 3508-05.2005. 